\title{
Lung clearance index in cystic fibrosis subjects treated for pulmonary exacerbations
}

\author{
Nicole Sonneveld ${ }^{1,15}$, Sanja Stanojevic ${ }^{1,2,3,15}$, Reshma Amin ${ }^{1,3}$, Paul Aurora ${ }^{4}$, \\ Jane Davies ${ }^{5}$, J. Stuart Elborn ${ }^{6}$, Alex Horsley ${ }^{7}$, Philipp Latzin ${ }^{8,9}$, \\ Katherine 0'Neill ${ }^{6}$, Paul Robinson ${ }^{10,11}$,Emma Scrase ${ }^{4}$, Hiran Selvadurai ${ }^{10,11}$, \\ Padmaja Subbarao ${ }^{1,2,3}$, Liam Welsh ${ }^{12,13}$, Sophie Yammine ${ }^{8,9}$ and Felix Ratjen ${ }^{1,2,14}$ \\ Affiliations: ${ }^{1}$ Division of Respiratory Medicine, Hospital for Sick Children, Toronto, ON, Canada. ${ }^{2}$ Physiology \\ and Experimental Medicine, Research Institute, Hospital for Sick Children, Toronto, ON, Canada. ${ }^{3}$ Institute of \\ Health Policy, Management and Evaluation, University of Toronto, Toronto, ON, Canada. ${ }^{4}$ Great Ormond Street \\ Hospital, London, UK. ${ }^{5}$ Royal Brompton Hospital, London, UK. ${ }^{6}$ Centre for Infection and Immunity, School of \\ Medicine, Dentistry and Biomedical Sciences, Queen's University Belfast, Belfast, UK. "Institute of \\ Inflammation and Repair, University of Manchester, Manchester, UK. ${ }^{8}$ University Children's Hospital Bern, \\ Bern, Switzerland. ${ }^{9}$ University Children's Hospital Basel (UKBB), Basel, Switzerland. ${ }^{10}$ Dept of Respiratory \\ Medicine, The Children's Hospital at Westmead, Sydney, Australia. ${ }^{11}$ Discipline of Paediatrics and Child \\ Health, Sydney Medical School, University of Sydney, Sydney, Australia. ${ }^{12}$ Royal Children's Hospital, \\ Melbourne, Australia. ${ }^{13}$ Murdoch Children's Research Institute, Melbourne, Australia. ${ }^{14}$ Faculty of Medicine, \\ Dept of Paediatrics, University of Toronto, Toronto, ON, Canada. ${ }^{15}$ Both authors contributed equally.
}

Correspondence: Sanja Stanojevic, The Hospital for Sick Children, Respiratory Medicine, 555 University Ave Toronto, M5G 1X8, ON,Canada. E-mail: sanja.stanojeviclasickkids.ca

ABSTRACT Pulmonary exacerbations are important clinical events for cystic fibrosis (CF) patients. Studies assessing the ability of the lung clearance index (LCI) to detect treatment response for pulmonary exacerbations have yielded heterogeneous results. Here, we conduct a retrospective analysis of pooled LCI data to assess treatment with intravenous antibiotics for pulmonary exacerbations and to understand factors explaining the heterogeneous response.

A systematic literature search was performed to identify prospective observational studies. Factors predicting the relative change in LCI and spirometry were evaluated while adjusting for within-study clustering.

Six previously reported studies and one unpublished study, which included 176 pulmonary exacerbations in both paediatric and adult patients, were included. Overall, LCI significantly decreased by 0.40 units $(95 \%$ CI $-0.60--0.19, \mathrm{p}=0.004)$ or $2.5 \%$ following treatment. The relative change in LCI was significantly correlated with the relative change in forced expiratory volume in $1 \mathrm{~s} \mathrm{(FEV1),} \mathrm{but} \mathrm{results} \mathrm{were}$ discordant in $42.5 \%$ of subjects (80 out of 188). Higher (worse) baseline LCI was associated with a greater improvement in LCI (slope: $-0.9 \%, 95 \%$ CI $-1.0--0.4 \%$ ).

LCI response to therapy for pulmonary exacerbations is heterogeneous in CF patients; the overall effect size is small and results are often discordant with FEV1.

@ERSpublications

Lung clearance index response to therapy for pulmonary exacerbations is heterogeneous in cystic fibrosis patients http://ow.ly/Mnvtd 


\section{Introduction}

New treatments in cystic fibrosis (CF) have attenuated lung damage and many subjects now maintain their spirometric lung function within the normal range well into early adulthood [1]. Despite normal spirometric lung function there is frequently evidence of structural damage measured by high-resolution computed tomography $[2,3]$. In this new era of CF care, improvements in lung function mean that spirometry may no longer be sensitive enough to detect or monitor disease progression, which emphasises the need for more sensitive lung function outcomes.

The lung clearance index (LCI), measured by the multiple breath washout (MBW) test, is a lung function outcome that has been shown to be more sensitive than spirometry [4-10], to correlate with airway changes seen on high-resolution computed tomography [11-13] and to detect significant treatment effects in randomised controlled trials in children with mild lung disease (forced expiratory volume in $1 \mathrm{~s}$ (FEV1) $\%$ predicted $>80 \%$ ) [14-16]. In addition, it is feasible to perform the test in all age groups [7, 17-19], and there are now commercial devices available to facilitate testing in the clinical setting. Currently, less is known about the ability of LCI to detect treatment response in patients with moderate-to-severe lung disease; a population that is more likely to require hospitalisation for pulmonary exacerbations.

Pulmonary exacerbations are important events for subjects with CF; approximately half of FEV1 decline can be attributed to pulmonary exacerbations requiring intravenous antibiotics [20]. In addition, the number of pulmonary exacerbations in a year is associated with poorer 5-year survival [21] and worse health-related quality of life [22]. Given the importance of pulmonary exacerbations for patients with $\mathrm{CF}$ and the potential ability to detect treatment response of LCI as an outcome measure, several studies have investigated whether LCI can detect a treatment response to i.v. antibiotics used to treat a pulmonary exacerbation. In contrast to studies where FEV1 was used as the primary outcome, which consistently showed a positive treatment effect [23-26], studies using LCI as an outcome measure have yielded heterogeneous results $[13,27-30]$. The reasons why this is the case when LCI is used as the primary outcome measure are not well understood.

In this study we analysed available LCI data from studies investigating response to i.v. antibiotics for a pulmonary exacerbation to understand what factors may explain the heterogeneity of the LCI response.

\section{Methods}

\section{Literature search}

A MEDLINE search was conducted in March 2014 to identify published studies that used MBW to measure response to i.v. antibiotics for pulmonary exacerbations using the following search terms: (LCI; lung clearance index; MBW; multiple breath washout; ventilation inhomogeneity; index of ventilation; inert gas washout; $\mathrm{SF}_{6}$; sulphur hexafluoride; $\mathrm{N}_{2}$; nitrogen washout) and (pulmonary exacerbation; acute exacerbation; antibiotic; antibiotics). Conference abstracts from January 2006 to March 2014 from the American Thoracic Society conference, the European Respiratory Society congress, the North American CF Conference and the European CF Conference were also searched for the same keywords. To be included in these analyses studies were limited to those conducted in subjects with CF with documented treatment with i.v. antibiotics for a pulmonary exacerbation (administered in a hospital or at home), and at least two measurements of MBW and spirometry (i.e. at the start ( $\pm 72 \mathrm{~h}$ ) and end of treatment). To be included in this study, patients had to be treated for $\geqslant 7$ days. MBW had to be measured in triplicate at each test occasion, with at least two acceptable trials and a valid LCI result at each test occasion.

\section{Data extraction}

The primary or senior author(s) of each of the eligible studies were contacted and invited to share data. The following explanatory variables were collected: age, sex, height, weight, body mass index, treatment duration, pancreatic status, cystic fibrosis-related diabetes, duration of hospitalisation and microbiology in the previous year (Pseudomonas aeruginosa, Burkholderia cepacia, (methicillin resistant) Staphylococcus aureus, Haemophilus influenzae, allergic bronchopulmonary aspergillosis (ABPA) and Stenotrophomonas maltophilia).

Support statement: The study reported by Horsley et al. was conducted by the UK CF Gene Therapy Consortium, and funded by a grant from the UK Cystic Fibrosis Trust. Alex Horsley is supported by a National Institute for Health Research (NIHR) award (NIHR-CS012-13); the views expressed are those of the author and not necessarily those of the UK NHS, the NIHR or the Department of Health. The study reported by O'Neil was funded by HSC (Health and Social Care) Research and Development, Public Health Agency Northern Ireland (UK) and the Medical Research Council through a USA-Ireland partnership grant. Funding information for this article has been deposited with FundRef.

Conflict of interest: Disclosures can be found alongside the online version of this article at erj.ersjournals.com 


\section{Statistical analysis}

Descriptive statistics were used to summarise the combined study population and each of the studies separately. Anthropometric data were converted to standardised scores using the Centers for Disease Control and Prevention 2000 reference equations. For adult subjects z-scores were calculated based on expected height and weight at the age of 19 years. FEV1 (L) measured by spirometry was converted to \% predicted using the Global Lungs Initiative 2012 reference equations [31].

Variables that predicted the relative change in LCI and FEV1 ((day 14 -day 0$) /$ day $0 \times 100)$ were evaluated using linear regression within a generalised estimating equation model to adjust for the correlated nature of the data arising within each study. The correlation between relative changes in LCI and FEV1 was assessed using a Pearson correlation.

In a cross-sectional analysis [32], results obtained from sulfur hexafluoride $\left(\mathrm{SF}_{6}\right)$ and nitrogen $\left(\mathrm{N}_{2}\right) \mathrm{MBW}$ methods have been shown to differ in individuals; it is presently unclear whether treatment responses also differ between these MBW methods. We analysed MBW results for each MBW method separately and as a combined dataset. In addition, we included an unpublished dataset from the Hospital for Sick Children (Toronto, Canada; see online supplementary material for details). Briefly, patients in the unpublished study were aged 6-18 years and were recruited on the day of their hospital admission for treatment with 2 weeks i.v. antibiotics for a pulmonary exacerbation. The choice of antibiotic was made by the admitting physician. MBW was measured within $48 \mathrm{~h}$ of the admission and discharge using both $\mathrm{SF}_{6}$ and $\mathrm{N}_{2} \mathrm{MBW}$ methods. Individual subject response between the methods was compared using Pearson correlation and a Bland-Altman plot.

There is currently no predefined cut-off value for a relative change in LCI, whereas several cut-offs have been proposed for the FEV1 [33-35]. Subjects were categorised into responders (improvement) and nonresponders (no response or deterioration) for LCI and FEV1, based on four cut-offs of relative change $(0 \%, 5 \%, 10 \%$ and $15 \%)$. We then investigated the agreement between LCI and FEV1 at each of these cut-off values using a $\kappa$-statistic. The proportion of discordant pairs was also reported. Statistical analysis was performed using Stata (version 12; StataCorp, College Station, TX, USA).

Each contributing study had research ethics board approval to collect data; in addition we obtained research ethics board approval from the Hospital for Sick Children (REB \#1000045313) to collate de-identified data from multiple studies.

\section{Results}

\section{Data collation and subject demographics}

The literature search identified 11 articles/conference abstracts representing seven studies (fig. 1) [8, 9, 13, 27-30, 36-39]. One study was excluded because of a methodology difference in MBW measurements,

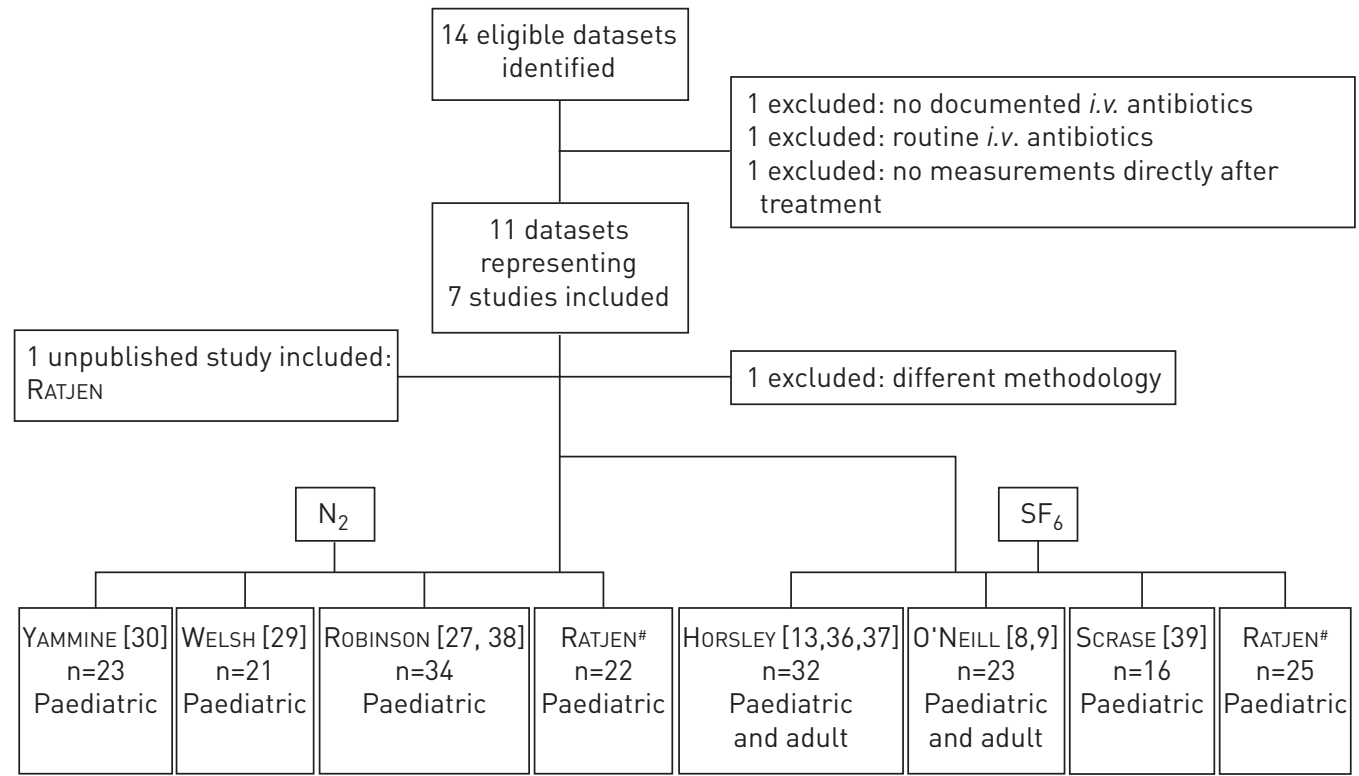

FIGURE 1 Flow chart of study selection and eligible studies that investigated the effects of intravenous antibiotics for a pulmonary exacerbation using multiple breath washout as an outcome in patients with cystic fibrosis. $\mathrm{n}$ indicates the original study population prior to any exclusions specific for this study. RATJEN conducted a single study where subjects were measured on both sulfur hexafluoride $\left(\mathrm{SF}_{6}\right)$ and nitrogen $\left(\mathrm{N}_{2}\right)_{\text {; }}$ 16 subjects had measurements on both devices. ${ }^{\#}$ : unpublished data. 
since tidal breathing was fixed to a standard volume [28]. Authors from all other studies were contacted and contributed data to the analysis. One unpublished study performed at the Hospital for Sick Children, which included a subgroup of 16 subjects with $\mathrm{MBW}$ measured on both the $\mathrm{N}_{2}$ and $\mathrm{SF}_{6}$ systems was also included. 10 pulmonary exacerbation events were excluded from the overall dataset because the time between the two MBW measurements was $<7$ days. In total, data from 176 pulmonary exacerbations from 172 subjects were included in these analyses.

The study population included five paediatric datasets and two studies with both adult and paediatric data (table 1). Upon admission the median (range) LCI was 13.7 units (7.7-21.7 units), and FEV1 was $55.7 \%$ predicted (range $17.9-105.4 \%$ predicted). The treatment duration was 14 days (8-31 days) and the time between MBW measurements was 12 days (7-29 days). Baseline FEV1 was greater in the $\mathrm{N}_{2}$ studies compared with the $\mathrm{SF}_{6}$ studies, whereas the absolute and relative FEV1 change did not differ between the two MBW methods (table 2).

\section{Physiological response to i.v. antibiotics}

Overall LCI significantly decreased by 0.40 units (95\% CI $-0.60--0.19, \mathrm{p}=0.004)$ or by $2.5 \%$ following treatment with i.v. antibiotics (table 2). LCI decreased by 0.49 units ( $95 \% \mathrm{CI}-0.78--0.20)$ or $3 \%$ in the four $\mathrm{SF}_{6}$ studies and by 0.31 units $(95 \% \mathrm{CI}-0.60--0.02)$ or $2 \%$ in the four $\mathrm{N}_{2}$ studies (fig. 2). The greater decline in the $\mathrm{SF}_{6}$ studies was driven by one study where the LCI decreased by -1.27 units; changes in FEV1 were also more pronounced in this study $[8,9]$. The average slope of the $\mathrm{N}_{2}$ and $\mathrm{SF}_{6}$ decline was similar when this study was excluded from the analysis $\left(\mathrm{SF}_{6}-0.33,95 \% \mathrm{CI}-0.66-0.00\right.$; $\mathrm{N}_{2}-0.31,95 \%$ CI $\left.-0.60--0.02\right)$. There was fair agreement for the relative change in LCI between the two systems in the 16 subjects who had both $\mathrm{MBWSF}_{6}$ and $\mathrm{MBWN}_{2}$ measured on the same day (mean difference -0.02 , 95\% CI $-5.5-5.5$; limits of agreement $-20.6-20.5$ ).

FEV1 increased by $14 \%$ (95\% CI $10-17 \%$ ) overall; by $18 \%$ in the $\mathrm{SF}_{6}$ studies and $10 \%$ in the $\mathrm{N}_{2}$ studies. Similar to LCI measures, the greater improvement for $\mathrm{SF}_{6}$ was driven by one dataset, where FEV 1 and LCI changes were more pronounced.

The relative change in LCI was weakly but significantly correlated with the relative change in FEV1 (fig. 3; Pearson correlation coefficient $-0.316, \mathrm{p}<0.0001)$. LCIsF $_{6}$ data had a stronger correlation with FEV1 $(\mathrm{R}=-0.408)$ than $\mathrm{LCIN}_{2}(\mathrm{R}=-0.212)$. The correlation analysis does not consider the clustering within centres, and therefore the coefficient may be overestimated. Comparison of different cut-offs for relative improvement between LCI and FEV1 demonstrated lack of agreement $(\kappa<0.25)$ between LCI and FEV1 (table 3). For each of the cut-offs, more subjects experienced an improvement for FEV1 than for LCI. When a $10 \%$ cut-off for FEV1 and a $-10 \%$ cut-off for LCI was used (fig. 3), there were 80 discordant pairs, of which 61 improved for FEV1, but not for LCI, and 19 improved for LCI but not FEV1 (fig. 3).

LCI is the cumulative expiratory volume (CEV) divided by functional residual capacity (FRC). FRC measured by MBW did not change with treatment (mean difference 0.9\%, 95\% CI -1.3\%-3.1\%). However, a negative correlation was observed between changes in FRC and changes in LCI, such that FRC increased when LCI improved $(\mathrm{R}=-0.329, \mathrm{p}<0.001)$. In a subset of the subjects where FEV1 improved (by $>10 \%)$ but LCI did not (>-10\%), FRC did not change $(1.8 \mathrm{~mL}, 0.009 \%)$. Conversely, in the subjects where FEV1 did not improve but LCI did, the FRC increased by $84 \mathrm{~mL}(0.04 \%)$. The CEV responded in the opposite direction for these dichotomous pairs, increasing by $1.1 \%$ in the group where FEV 1 improved and LCI did not and decreasing by $7.7 \%$ in the group where FEV1 did not improve and LCI did. FRC and CEV behaved similarly in the $\mathrm{N}_{2}$ and $\mathrm{SF}_{6}$ studies and in the patients that were measured on both systems.

\section{Predictors of change in $\mathrm{LCl}$ and FEV 1}

Higher (worse) baseline (day 0) LCI values were associated with a greater improvement in LCI (slope $-0.9 \%$, 95\% CI $-1.0--0.4 \%$ ) (fig. 4). Similarly, worse FEV1 at baseline was associated with greater improvement of FEV1. Longer treatment duration was associated with smaller improvements in both LCI and FEV1. In the paediatric data, a higher height-for-age z-score (slope $-2.0 \%, 95 \%$ CI $-4.0--0.9 \%$ ) and a higher weight-for-age z-score (slope $-3.0 \%, 95 \%$ CI $-4.0 \%--0.7 \%$ ) were associated with greater improvements in LCI, but not FEV1.

In the subset with available data a recent infection with $S$. maltophilia (within the last year) was the only microbiological variable associated with a poorer FEV1 response (fig. 4).

A sensitivity analysis excluding 14 subjects with ABPA in the last 12 months showed the same associations as the complete dataset (data not shown). The univariate analyses were also repeated for the two MBW methods separately. The predictors from the stratified analyses were similar to those when the MBW methods were combined (data not shown). 
TABLE 1 Summary of population characteristics for the combined study population and each of the seven datasets separately

\begin{tabular}{|c|c|c|c|c|c|c|c|c|}
\hline & Combined & Horsley $[13,36,37]$ & O'Neill $[8,9]$ & RATJEN ${ }^{\#}$ & Robinson $[27,38]$ & SCRASE [39] & WeLsh [29] & Yammine [30] \\
\hline Exacerbations $\mathbf{n}$ & 176 & 32 & 21 & 31 & 34 & 16 & 21 & 21 \\
\hline Age at admission years & $15(5-56)$ & $23(11-44)$ & $24(12-56)$ & $14(7-18)$ & $14(8-17)$ & $11(8-17)$ & $17(9-20)$ & $13(5-19)$ \\
\hline Male & $76(43.2)$ & $20(62.50)$ & $10(47.6)$ & $11(35.5)$ & 12 (35.3) & 5 (31.3) & 9 (42.9) & $9(42.9)$ \\
\hline $\mathrm{LCl}$ at admission & $12.5(6.9-21.7)$ & $15.0(10.7-21.7)$ & $11.9(7.7-19.8)$ & $12.4(9.1-19.0)^{\pi}$ & $9.9(6.9-14.8)$ & $14.0(10.2-18.8)$ & $12.8(8.4-17.9)$ & $13.7(8.3-19.4)$ \\
\hline FEV 1 at admission $\%$ pred & $59.2(17.9-105.4)$ & $53.2(32.1-86.2)$ & $45.4(17.9-93.4)$ & $59.2(28.4-103.1)$ & $67.2(25.1-95.4)$ & $48.0(22.6-105.4)$ & $64.1(18.6-95.1)$ & $74.1(54.2-104.6)$ \\
\hline Treatment duration days & $14(8-31)$ & 14 (9-22) & $15(8-20)$ & $14(11-31)$ & $15(12-20)$ & $\mathrm{N} / \mathrm{A}$ & $13(8-18)$ & $14(10-28)$ \\
\hline Time between tests days & $12(7-29)$ & $13(7-21)$ & $13(7-16)$ & $12(8-16)$ & $13(9-14)$ & $11.5(8-18)$ & $10(7-14)$ & $13(7-29)$ \\
\hline \multicolumn{9}{|l|}{ Complications } \\
\hline Pancreatic insufficiency & $151(94.4)$ & $29(90.6)$ & $21(100)$ & $29(93.6)$ & 33 (97.1) & N/A & 18 (85.7) & $21(100)$ \\
\hline CFRD & 25 (18.0) & $11(34.4)$ & $4(19.1)$ & $2(6.5)$ & 5 (14.7) & $\mathrm{N} / \mathrm{A}$ & $\mathrm{N} / \mathrm{A}$ & $3(14.3)$ \\
\hline ABPA & 14 (10.53) & $1(3.1)$ & 0 & 1 (3.3) & $6(17.6)$ & $\mathrm{N} / \mathrm{A}$ & $\mathrm{N} / \mathrm{A}$ & $6(28.6)$ \\
\hline \multicolumn{9}{|l|}{ Microbiology } \\
\hline P. aeruginosa & $89(64.0)$ & $21(65.6)$ & 13 (81.3) & $18(58.1)$ & $33(97.1)$ & N/A & $6(28.6)$ & $11(52.4)$ \\
\hline S. aureus & 78 (66.7) & $16(50.0)$ & 9 (56.3) & $26(86.7)$ & 18 (52.9) & $\mathrm{N} / \mathrm{A}$ & N/A & 18 (85.7) \\
\hline MRSA & $8(6.8)$ & $3(9.4)$ & $1(6.3)$ & $2(6.5)$ & $3(8.8)$ & N/A & N/A & $0(0)$ \\
\hline H. influenzae & $32(27.4)$ & $5(15.6)$ & $1(6.3)$ & $15(50.0)$ & $3(8.8)$ & N/A & N/A & $9(42.9)$ \\
\hline B. cepacia complex & $10(8.5)$ & 7 (21.9) & $2(12.5)$ & $0(0)$ & $2(5.9)$ & N/A & N/A & $1(4.8)$ \\
\hline S. maltophilia & $30(25.6)$ & $7(21.9)$ & $2(12.5)$ & $11(36.7)$ & $2(5.9)$ & N/A & N/A & $10(47.6)$ \\
\hline
\end{tabular}

Data are presented as median (range) or $\mathrm{n}(\%)$, unless otherwise stated. LCl: lung clearance index; FEV1: forced expiratory volume in $1 \mathrm{~s}$; \% pred: \% predicted; CFRD: cystic fibrosis-related diabetes; ABPA: allergic bronchopulmonary aspergillosis; P. aeruginosa: Pseudomonas aeruginosa; S. aureus: Staphylococcus aureus; MRSA: methicillin-resistant S. aureus; H. influenzae: Haemophilus influenzae; B. cepacia: Burkholderia cepacia; S. maltophilia: Stenotrophomonas maltophilia; N/A: not available. \#: unpublished data. П: LCI measured using both sulfur hexafluoride $\left(\mathrm{SF}_{6}\right)$ and nitrogen $\left(\mathrm{N}_{2}\right)$ in 16 subjects; $\mathrm{LCl}$ is presented from the subjects that completed $\mathrm{SF}_{6}$ measurements; the corresponding median (range) for the $\mathrm{N}_{2}$ measurements was $14.8(9.2-22.2)$ 
TABLE 2 Changes in lung clearance index (LCI) and forced expiratory volume in $1 \mathrm{~s}$ (FEV1) for the overall study population and for sulfur hexafluoride $\left(\mathrm{SF}_{6}\right)$ and nitrogen $\left(\mathrm{N}_{2}\right)$ multiple breath washout (MBW) studies separately

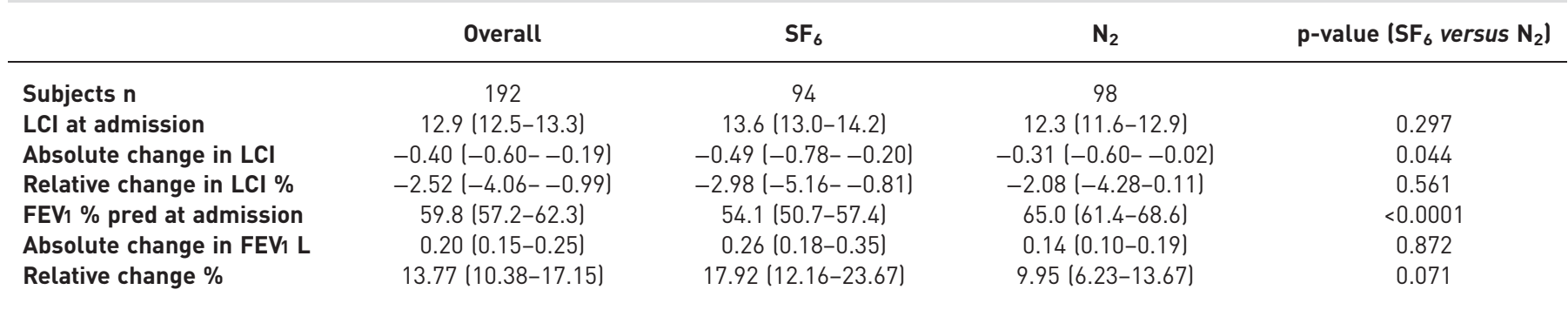

Data are presented as mean $(95 \% \mathrm{Cl})$, unless otherwise stated. p-values were generated using linear regression with tracer gas as an explanatory variable adjusting for the correlated nature of the data using generalised estimating equation models.

\section{Discussion}

In this analysis of pooled data we assessed whether the LCI can be used to assess treatment response in patients receiving i.v. antibiotic therapy for pulmonary exacerbations. Overall, LCI decreased significantly with treatment regardless of whether the methodology used was $\mathrm{SF}_{6}$ - or $\mathrm{N}_{2}$-based, but the effect size was small. Changes in LCI were weakly correlated to changes in FEV1 and discordant results were observed in a significant proportion of subjects.

While the changes in LCI were statistically significant, the overall effect size was smaller than for FEV 1 and smaller than previously reported for interventional studies assessing treatment effects on LCI of agents that promote clearance of airway secretions (hypertonic saline [14] and dornase alfa [40]) and the CF transmembrane conductance regulator modulator ivacaftor [15]). While a minimal clinically important difference has not been clearly defined in these studies, the overall change is less than the inter-visit variability in the placebo groups of these previous studies. Several factors may account for this difference. First, previous interventional studies have been conducted over 4-week intervals, while the median duration of treatment for pulmonary exacerbations in this study was 2 weeks. The time course of treatment response of LCI is not well established; however, the recent ivacaftor study [15] demonstrated that the treatment effect reached its maximum at the 2-week time point. These interventional drug studies have focused on patients with mild lung function ( $\mathrm{FEV} 1>80 \%$ pred) and sensitivity of LCI may vary with disease severity. Interestingly, we observed that higher LCI (reflecting worse lung function) was associated with larger relative improvement, which would go against the hypothesis that LCI may be less suitable in patients with more severe disease. Lastly, differences in the ways that these interventions improve lung function may explain the observed differences. Hypertonic saline, dornase alfa and ivacaftor all improve mucus clearance from the airways, while antibiotic therapy mainly targets infection and inflammation. Nonetheless, the retrospective nature of these analyses mean that the intervention was not standardised, and patients included in these analyses may have been on additional treatments such as systemic steroids and intensified mucus clearance regimes.

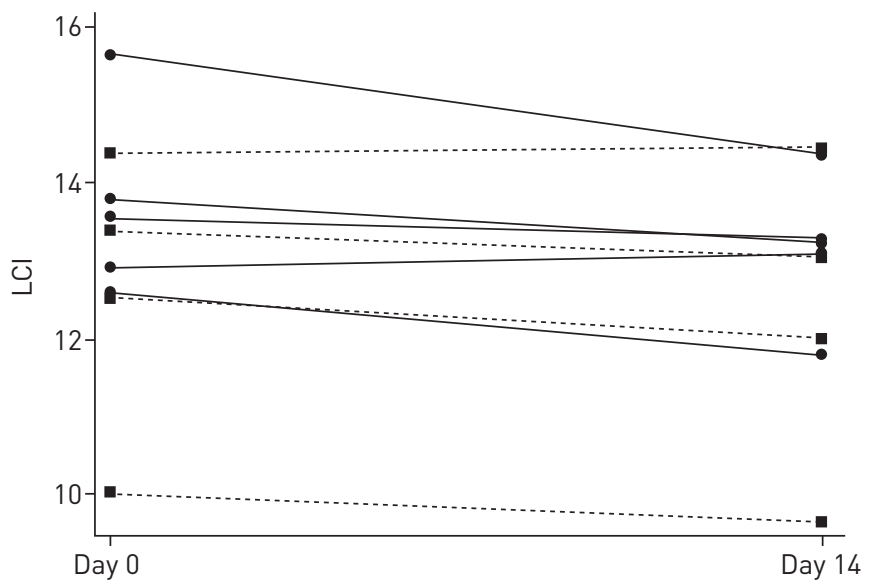

FIGURE 2 Mean change in lung clearance index (LCI) from day 0 to day 14 per study. Solid lines represent studies that used sulfur hexafluoride as a tracer gas; dashed lines represent studies that used nitrogen as the tracer gas. 


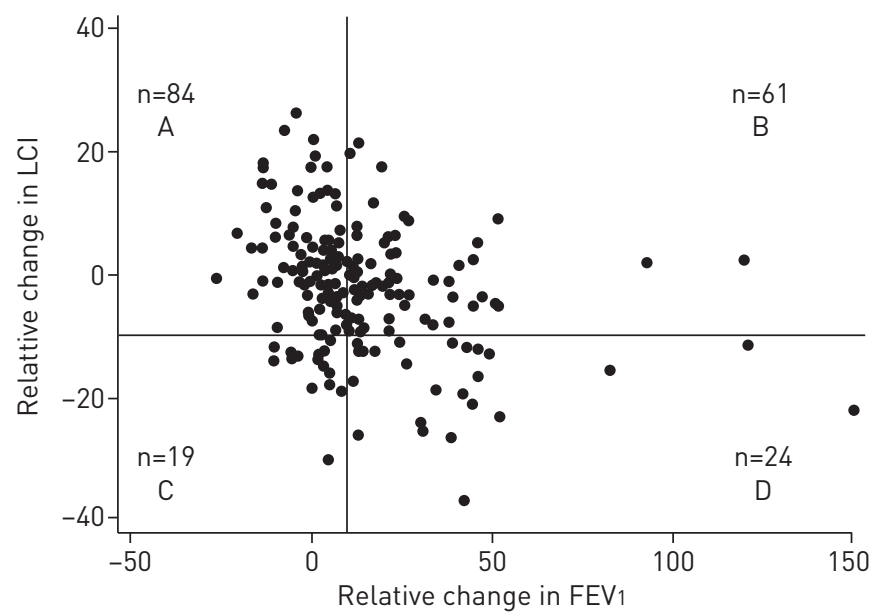

FIGURE 3 Correlation analysis between the relative changes in lung clearance index (LCI) and forced expiratory volume in $1 \mathrm{~s}$ ( $F E V_{1}$ ) (Pearson correlation coefficient $-0.316, p<0.0001$ ), demonstrates the discordance between the two lung function outcomes. $\mathrm{A}$ : deterioration in $\mathrm{LCl}$ and $\mathrm{FEV} 1 ; \mathrm{B}$ : improvement in $\mathrm{FEV} 1$ but not $\mathrm{LCl} ; \mathrm{C}$ : improvement in $\mathrm{LCl}$ but not $\mathrm{FEV}$; and $\mathrm{D}$ : improvement in both $\mathrm{LCl}$ and $\mathrm{FEV} 1$ Among the discordant results, 19 subjects had an improvement of their $\mathrm{LCl}$, but not $\mathrm{FEV}_{1}$, whereas 61 had an improvement in their $\mathrm{FEV}_{1}$, but not in LCl. The correlated nature of data within centres is not accounted for in these correlation analyses, and therefore the estimates may be biased.

We observed a significant proportion of discordant pairs in detecting treatment success depending on the outcome used (i.e. LCI versus FEV1), regardless of which cut-off was chosen. LCI and FEV1 measure different aspect of lung physiology, therefore this finding is not surprising per se. FEV1 mainly reflects large airway function and will be affected by changes in airway tone, mucus accumulation in the airways and air trapping. Previous computed tomography studies have demonstrated that all of these factors can improve with treatment of a pulmonary exacerbation, but that the predominant component may vary between patients $[12,13]$. Recent studies would suggest that mucus plugging, and specifically large-airway mucus plugging changes more than other aspects of CF lung disease with antibiotic therapy [12, 41]. In contrast, LCI is affected by heterogeneities at all levels of the airway tree, including peripheral airway disease, and changes in bronchomotor tone do not affect the measurement as much as FEV 1 , as demonstrated by studying LCI before and after administration of bronchodilators [42-44]. Previously closed compartments contributing to the measurement could also affect LCI response. Overall, we did not observe any changes in FRC measured by MBW which would argue against the hypothesis that that previously closed compartments affect LCI through changes in FRC. In fact, deterioration in LCI was weakly correlated with decrease in FRC suggesting increases in trapped gas and/or increased hyperinflation in patients where LCI increased despite treatment. YAmmine et al. [30] investigated the physiological mechanisms behind the heterogeneous response to treatment in a smaller group of subjects. They reported that a measurement of trapped gas (FRCMBW minus the residual volume measured by plethysmography) together with ventilation homogeneity explained $58 \%$ of the variability of the change in LCI, suggesting that improvements in LCI might be a result of less secretion and airway obstruction, better ventilated lung

TABLE 3 Agreement between lung clearance index (LCI) and forced expiratory volume in $1 \mathrm{~s}$ (FEV 1 ) to identify subjects with an improvement in each outcome according to four different cut-offs

\begin{tabular}{cccccc} 
& $\begin{array}{c}\text { Subjects with an } \\
\text { improvement in } \\
\text { outcome }\end{array}$ & $\mathbf{0 \%}$ & $\mathbf{5 \%}$ & $\mathbf{1 0 \%}$ & $\mathbf{1 5 \%}$ \\
\cline { 3 - 6 } & & $112(59.6)$ & $72(38.3)$ & $\mathbf{4 3}(22.9)$ & $19(10.1)$ \\
Relative improvement in FEV & $145(77.1)$ & 34.6 & 47.3 & 59.6 & 67.0 \\
$0 \%$ & $115(61.2)$ & 36.7 & 44.1 & 52.1 & 53.1 \\
$5 \%$ & $85(45.2)$ & 38.9 & 41.0 & 42.6 & 41.5 \\
$10 \%$ & $59(31.4)$ & 46.2 & 59.6 & 34.0 & 29.8 \\
$15 \%$ & & & & &
\end{tabular}

Data are presented as $\mathrm{n}(\%)$ or $\%$. The proportion of subjects above each of the cut-offs is presented as $n(\%)$, as well as the percentage of discordant pairs. $\kappa<0.25$ for all of the comparisons, indicating poor agreement. 
a)

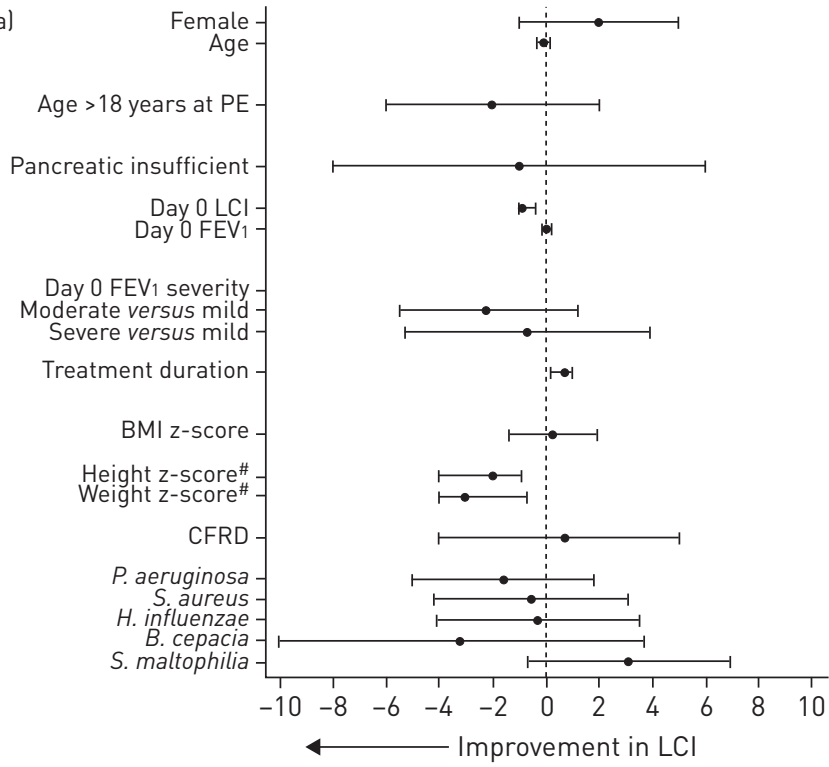

b)

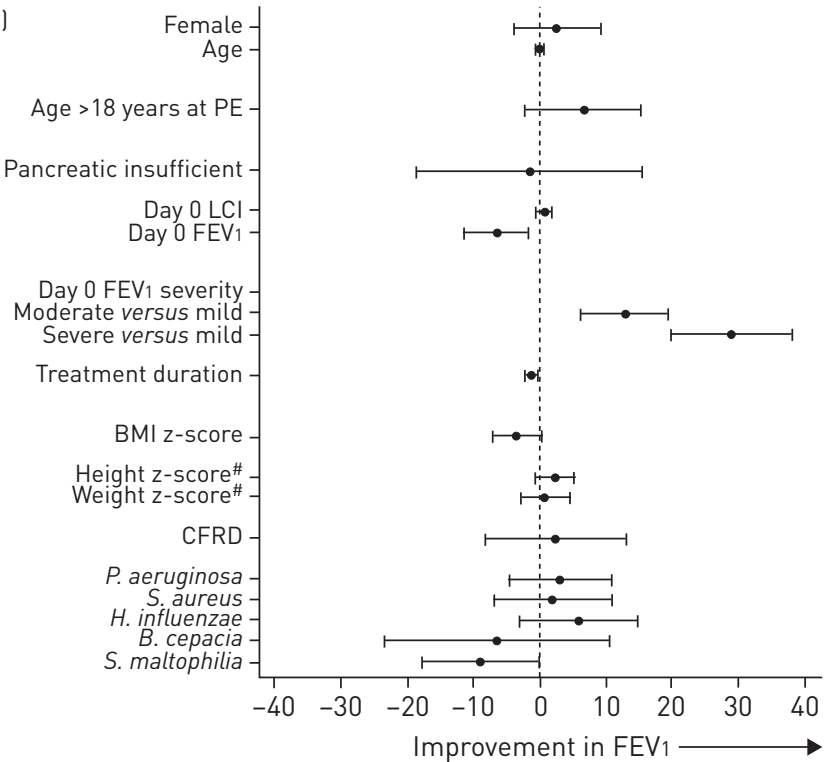

FIGURE 4 a) Univariate predictors of lung clearance index (LCI) change following 2 weeks of intravenous antibiotic treatment. A negative change in $\mathrm{LCl}$ indicates that patients improved following treatment. For continuous variables lage, day $0 \mathrm{LCl}$, day 0 forced expiratory volume in $1 \mathrm{~s}$ (FEV 1 ), treatment duration and nutritional z-scores) results correspond to the average LCl change following treatment for each 1-unit increase in each continuous variable. b) Univariate predictors of FEV1 change following 2 weeks of i.v. antibiotic treatment. A positive change in FEV 1 indicates that patients improved following treatment. For continuous variables (age, day 0 LCI, day 0 FEV1, treatment duration and nutritional z-scores) results correspond to the average FEV1 change following treatment for each 1-unit increase in each continuous variable. PE: pulmonary exacerbation; BMI: body mass index; CFRD: cystic fibrosis-related diabetes; P. aeruginosa: Pseudomonas aeruginosa; S. aureus: Staphylococcus aureus; H. influenzae: Haemophilus influenzae; B. cepacia: Burkholderia cepacia; S. maltophilia: Stenotrophomonas maltophilia. ": calculated for paediatric patients only.

units and less hyperinflation [30]. Parallel measurements of lung volume by body plethysmography were not available for most studies and therefore we could not explore this relationship with the current dataset.

Evaluation of the predictors of the change in LCI demonstrated that worse ventilation inhomogeneity (higher LCI values) at the start of an exacerbation resulted in greater improvement in LCI. This is in contrast with the assumption that MBW might be a less useful outcome measure in more advanced disease. We also observed that better nutrition in paediatric subjects was associated with greater improvement in LCI. This is unlikely to be causally related to treatment response, but rather mediated through the health status of the subject. Longer treatment duration was associated with a poorer response for LCI and FEV1, but this may be a proxy for a lack of clinically perceived response resulting in prolongation of therapy, rather than being causally related. While these factors contributed to the variability of response, they did not sufficiently explain the discordance in treatment response between LCI and FEV1. Further evidence from longitudinal data is needed to clarify how these discrepancies in treatment response are linked to clinical outcomes of patients.

\section{Strengths and limitations}

In this analysis we pooled several datasets to investigate whether LCI can be used to assess treatment response to i.v. antibiotics for a pulmonary exacerbation in subjects with CF. Results were similar when data were stratified by MBW method. However, the retrospective nature limits these analyses in that each of the included studies were designed and conducted independently, using different definitions of a pulmonary exacerbation and applying different treatment protocols. While we adjusted for the correlated nature of data from the same study in the statistical analyses by applying population average methods for clustered data, the differences in protocols and study populations may have introduced bias into the estimates and associations. Furthermore, these analyses were limited to the predictor variables available for each of the studies, which was not an exhaustive list of potential factors that may have influenced the response to treatment. The different equipment and MBW methods used also meant that not all MBW outcomes could be investigated (e.g. moment ratios, Scond and Sacin). Current practice defines treatment success based on whether subjects' FEV1 values return to $90 \%$ of their stable baseline values [25]; our retrospective analyses of pooled data were limited since pre-exacerbation baseline values were not available. Finally, we observed a statistically significant improvement in LCI with treatment with i.v. antibiotics; whether the observed magnitude of improvement is clinically meaningful is yet to be determined, since at present, there is no consensus regarding a minimal clinically important difference for LCI in CF subjects. 


\section{Conclusions}

In conclusion, this study demonstrates that LCI decreases significantly in patients treated for a pulmonary exacerbation. The overall effect size was smaller for LCI than for FEV1, and there was discordance between FEV1 and LCI. Future studies should assess how treatment response assessed by LCI is linked to the subsequent course of lung disease as well as long-term outcomes.

\section{References}

1 Cystic Fibrosis Foundation. Patient Registry 2012 Annual Data Report. Bethesda, CF Foundation, 2012.

2 Sly PD, Brennan S, Gangell C, et al. Lung disease at diagnosis in infants with cystic fibrosis detected by newborn screening. Am J Respir Crit Care Med 2009; 180: 146-152.

3 Tiddens HA. Detecting early structural lung damage in cystic fibrosis. Pediatr Pulmonol 2002; 34: 228-231.

4 Gustafsson PM, Aurora P, Lindblad A. Evaluation of ventilation maldistribution as an early indicator of lung disease in children with cystic fibrosis. Eur Respir J 2003; 22: 972-979.

5 Aurora P, Gustafsson P, Bush A, et al. Multiple breath inert gas washout as a measure of ventilation distribution in children with cystic fibrosis. Thorax 2004; 59: 1068-1073.

6 O'Neill K, Johnston E, Tunney MM, et al. Lung clearance index (LCI): a sensitive outcome measure in adults and children with cystic fibrosis (CF). Phys Ther Rev 2014; 19: 57.

7 Horsley AR, Gustafsson PM, Macleod KA, et al. Lung clearance index is a sensitive, repeatable and practical measure of airways disease in adults with cystic fibrosis. Thorax 2008; 63: 135-140.

8 O'Neill K, Elborn J, Johnston E, et al. Changes in lung clearance index during periods of stability and pulmonary exacerbation. Pediatr Pulmonol 2012; 47: Suppl. 35, 352-353.

9 O’Neill K, Johnston E, Tunney M, et al. Lung clearance index (LCI) during and following a pulmonary exacerbation (PEx) in patients with CF. J Cyst Fibros 2012; 11: Suppl. 1, S97.

10 Singer F, Kieninger E, Abbas C, et al. Practicability of nitrogen multiple-breath washout measurements in a pediatric cystic fibrosis outpatient setting. Pediatr Pulmonol 2013; 48: 739-746.

11 Owens CM, Aurora P, Stanojevic S, et al. Lung clearance index and HRCT are complementary markers of lung abnormalities in young children with CF. Thorax 2011; 66: 481-488.

12 Rowan SA, Bradley JM, Bradbury I, et al. Lung clearance index is a repeatable and sensitive indicator of radiological changes in bronchiectasis. Am J Respir Crit Care Med 2014; 189: 586-592.

13 Horsley AR, Davies JC, Gray RD, et al. Changes in physiological, functional and structural markers of cystic fibrosis lung disease with treatment of a pulmonary exacerbation. Thorax 2013; 68: 532-539.

14 Amin R, Subbarao P, Jabar A, et al. Hypertonic saline improves the LCI in paediatric patients with CF with normal lung function. Thorax 2010; 65: 379-383.

15 Davies J, Sheridan H, Bell N, et al. Assessment of clinical response to ivacaftor with lung clearance index in cystic fibrosis patients with a G551D-CFTR mutation and preserved spirometry: a randomised controlled trial. Lancet Respir Med 2013; 1: 630-638.

16 Subbarao P, Stanojevic S, Brown M, et al. Lung clearance index as an outcome measure for clinical trials in young children with cystic fibrosis. A pilot study using inhaled hypertonic saline. Am J Respir Crit Care Med 2013; 188: 456-460.

17 Stahl M, Joachim C, Blessing K, et al. Multiple breath washout is feasible in the clinical setting and detects abnormal lung function in infants and young children with cystic fibrosis. Respiration 2014; 87: $357-363$.

18 Lum S, Gustafsson P, Ljungberg H, et al. Early detection of cystic fibrosis lung disease: multiple-breath washout versus raised volume tests. Thorax 2007; 62: 341-347.

19 Kieninger E, Singer F, Fuchs O, et al. Long-term course of lung clearance index between infancy and school-age in cystic fibrosis subjects. J Cyst Fibros 2011; 10: 487-490.

20 Waters V, Stanojevic S, Atenafu EG, et al. Effect of pulmonary exacerbations on long-term lung function decline in cystic fibrosis. Eur Respir J 2012; 40: 61-66.

21 Liou TG, Adler FR, Fitzsimmons SC, et al. Predictive 5-year survivorship model of cystic fibrosis. Am J Epidemiol 2001; 153: 345-352.

22 Britto MT, Kotagal UR, Hornung RW, et al. Impact of recent pulmonary exacerbations on quality of life in patients with cystic fibrosis. Chest 2002; 121: 64-72.

23 Collaco JM, Green DM, Cutting GR, et al. Location and duration of treatment of cystic fibrosis respiratory exacerbations do not affect outcomes. Am J Respir Crit Care Med 2010; 182: 1137-1143.

24 Ordoñez CL, Henig NR, Mayer-Hamblett N, et al. Inflammatory and microbiologic markers in induced sputum after intravenous antibiotics in cystic fibrosis. Am J Respir Crit Care Med 2003; 168: 1471-1475.

25 Sanders DB, Bittner RC, Rosenfeld M, et al. Failure to recover to baseline pulmonary function after cystic fibrosis pulmonary exacerbation. Am J Respir Crit Care Med 2010; 182: 627-632.

26 VanDevanter DR, O'Riordan MA, Blumer JL, et al. Assessing time to pulmonary function benefit following antibiotic treatment of acute cystic fibrosis exacerbations. Respir Res 2010; 11: 137.

27 Robinson PD, Cooper P, Van Asperen P, et al. Using index of ventilation to assess response to treatment for acute pulmonary exacerbation in children with cystic fibrosis. Pediatr Pulmonol 2009; 44: 733-742.

28 Vanderhelst E, De Meirleir L, Schuermans D, et al. Evidence of an acinar response following treatment for exacerbation in adult patients with cystic fibrosis. Respiration 2014; 87: 492-498.

29 Welsh L, Nesci C, Tran H, et al. Lung clearance index during hospital admission in school-age children with cystic fibrosis. J Cyst Fibros 2014; 13: 687-691.

30 Yammine S, Bigler A, Casaulta C, et al. Reasons for heterogeneous change in LCI in children with cystic fibrosis after antibiotic treatment. Thorax 2014; 69: 183.

31 Quanjer PH, Stanojevic S, Cole TJ, et al. Multi-ethnic reference values for spirometry for the 3-95-yr age range: the global lung function 2012 equations. Eur Respir J 2012; 40: 1324-1343.

32 Jensen R, Stanojevic S, Gibney $\mathrm{K}$, et al. Multiple breath nitrogen washout: a feasible alternative to mass spectrometry. PloS One 2013; 8: e56868.

33 Griese M, Kappler M, Eismann C, et al. Inhalation treatment with glutathione in patients with cystic fibrosis. A randomized clinical trial. Am J Respir Crit Care Med 2013; 188: 83-89. 
34 Kerem E, Viviani L, Zolin A, et al. Factors associated with FEVı decline in cystic fibrosis: analysis of the ECFS patient registry. Eur Respir J 2014; 43: 125-133.

35 Retsch-Bogart GZ, Burns JL, Otto KL, et al. A phase 2 study of aztreonam lysine for inhalation to treat patients with cystic fibrosis and Pseudomonas aeruginosa infection. Pediatr Pulmonol 2008; 43: 47-58.

36 Horsley A, Macleod K, Saunders C, et al. UK CF Gene Therapy Consortium Tracking Study: lung clearance index improves with treatment of an infective exacerbation. Pediatr Pulmonol 2007; 42: Suppl. 30, 335-336.

37 Horsley A, Saunders C, Gray R, et al. Lung clearance index improves with treatment of an exacerbation in cystic fibrosis. Eur Respir J 2007; 30: Suppl. 51, S450.

38 Robinson P, Van Asperen P, Cooper P, et al. Assessing response to intravenous therapy for pulmonary exacerbations in children with cystic fibrosis. Pediatr Pulmonol 2006; 41: Suppl. 29, 348.

39 Scrase E, Oliver C, Kozlowska W, et al. Change in lung clearance index (LCI) following intravenous antibiotic intervention in children with CF. Eur Respir J 2008; 32: Suppl. 52, S514.

40 Amin R, Subbarao P, Lou W, et al. The effect of dornase alfa on ventilation inhomogeneity in patients with cystic fibrosis. Eur Respir J 2011; 37: 806-812.

41 Amin R, Charron M, Grinblat L, et al. Cystic fibrosis: detecting changes in airway inflammation with FDG PET/ CT. Radiology 2012; 264: 868-875.

42 Gustafsson PM. Peripheral airway involvement in CF and asthma compared by inert gas washout. Pediatr Pulmonol 2007; 42: 168-176.

43 Sonnappa S, Bastardo CM, Wade A, et al. Repeatability and bronchodilator reversibility of lung function in young children. Eur Respir J 2013; 42: 116-124.

44 Zwitserloot A, Fuchs SI, Muller C, et al. Clinical application of inert gas multiple breath washout in children and adolescents with asthma. Respir Med 2014; 108: 1254-1259. 